\title{
QUALE LOGICA (PER LA FISICA)?
}

\author{
MARCO ERBA (*)
}

\begin{abstract}
SuNTO. - La motivazione preminente alla pratica scientifica può essere ravvisata - in una sua formulazione debole - nell'individuazione di fenomeni ricorrenti, nonché nella loro manipolazione sperimentale e teorica. Si pone dunque il problema della necessità di adottare un sistema di regole per il processo logico di inferenza, al fine di assegnare una sintattica, un contenuto semantico, ed eventualmente un'interpretazione, alle evidenze empiriche. Secondo Aristotele, la non-contraddizione è "il più sicuro di tutti i princìpi": irrefutabile, pena l'impossibilità finanche di formulare pensieri. Nella presente relazione verranno esposte le implicazioni del rifiuto di alcune leggi della logica classica, come il principio di non contraddizione. Tale possibilità dà luogo ad una pluralità di sistemi logici, di cui verranno presi in esame alcuni aspetti significativi per la Matematica e per la Fisica. Si discuterà dunque di come si possa indirizzare la scelta di un sistema logico da adottare nella pratica scientifica. Il peculiare caso concreto della Teoria Quantistica verrà usato come fil rouge nel dipanarsi delle problematiche relazionate.
\end{abstract}

$* * *$

ABSTRACT. - The preeminent motivation to the scientific practice - stated in a weak way - can be recognized in the individuation of recurring phenomena (or else empirical regularity), along with the manipulation, both experimental and theoretical, of these. One can thus pose the issue of the necessity of adopting a set of rules for the logical inferential process, in order to assign a syntax, a semantic content, and possibly an interpretation, to the empirical evidences. According to Aristotle, non-contradiction is "the firmest principle of all": irrefutable, otherwise the very possibility of formulating thoughts fails. Throughout the present report, the entailments of refusing some of the laws of classical logic - e.g. non-contradiction - are exposed. Such a possibility sheds light on a plurality of logical systems: some traits of these, which are significant for Mathematics and Physics, are examined. For instance, the relevance of dialetheism and intuitionism will be discussed. Besides, the report discusses on which basis one should choose the logical system to be adopted for the scientific activity. The peculiar case study given by Quantum Theory serves as fil rouge in developing the reported matters.

(*) Postdoc, QUit Group, Dipartimento di Fisica, Università degli Studi di Pavia, Italia. E-mail: marco.erba@unipv.it 


\section{Il RUOLO DELLA LOGICA NELLA SCIENZA}

La pratica scientifica, lato sensu, si propone di: (a) rintracciare fenomeni ricorrenti al sussistere di fissate condizioni sperimentali; (b) modellare tale regolarità empirica all'interno di un formalismo quantitativomatematico; (c) poter formulare proposizioni che descrivano la preparazione di condizioni sperimentali e che siano dotate di potere predittivo.

Ognuno dei tre movimenti summenzionati, all'interno dei quali l'attività scientifica si sviluppa, presuppone l'istituzione di un sistema coerente di regole formali che permettano non solo di formulare proposizioni, ma anche di connetterle vicendevolmente al fine di inferire conclusioni ben formate. È dunque necessario un sistema logico dotato di operatori, assiomi e regole di inferenza: gli elementi teorici sono così posti in mutua relazione attraverso una sintattica. Infine, affinché il sistema logico e l'evidenza empirica possano essere tra loro relati, una teoria scientifica deve inoltre sussumere gli enti del proprio discorso sotto un modello. Il ruolo di quest'ultimo è di stabilire una semantica che permetta di manipolare i segni in modo da stabilire una ben posta corrispondenza tra asserti teorici e fenomeni osservati.

Diamo adesso una esemplificazione di quanto esposto finora, avvalendoci di un semplice esperimento mentale.

\subsection{Gedankenexperiment del mentitore}

Il lettore si figuri d'esser uno sperimentatore che allestisca un apparato sperimentale composto come di seguito. Si danno due bottoni, un registro di memoria contenente coppie di numeri, uno schermo e infine un soggetto che vi possa interagire. Ad ogni sessione dell'esperimento, l'apparato legge la coppia di numeri casuali associata e momentaneamente non la mostra al soggetto: lo schermo gli svelerà tanti numeri quanti saranno i tasti che questo decida di schiacciare, dunque rispettivamente: due, uno o nessuno. A questo punto al soggetto viene chiesto di interagire con l'apparato sperimentale un certo numero di volte; queste interazioni non sono poste sotto l'osservazione dello sperimentatore e inoltre il soggetto ha la possibilità di annotare ciò che ritenga ad ogni sessione, per poi riportare tutto allo sperimentatore. Ogni volta che l'esperimento viene condotto, il soggetto riporta correttamente l'intera coppia di numeri e inoltre annota: "Non ho schiacciato entrambi i tasti". Denotiamo quest'ultima affermazione con $\neg \mathrm{A}$, ove $\neg$ simboleggia l'operatore logico di 
negazione e A la proposizione "è il caso che io abbia schiacciato entrambi i tasti". Come in qualsiasi rilevazione sperimentale di fenomeni, si pone ora il problema di interpretare i risultati dell'esperimento descritto. Si dia il caso in cui siamo convinti che le condizioni sperimentali siano sotto il nostro controllo, cioè in particolare che il soggetto non possa aver accesso al registro di memoria senza schiacciare i tasti. Su una base statistica, avendo ripetuto l'esperimento per un numero grande di sessioni, riteniamo inoltre che il soggetto non possa aver tirato ad indovinare. Dunque, secondo il modello dell'apparato sperimentale allestito, riteniamo che sia impossibile aver avuto accesso ad entrambi i numeri senza aver schiacciato entrambi i tasti. Restringiamoci a questa eventualità: formuliamo la nostra credenza con l'ipotesi che $(\neg \neg \mathrm{A} \vee \neg \mathrm{B})$, ove B sta per "è il caso che il soggetto conosca entrambi i numeri” e $\vee$ simboleggia l'operatore logico di disgiunzione ("oppure"). Tale disgiunzione ipotetica si può formulare come "non è il caso che il soggetto non abbia schiacciato entrambi i tasti oppure non è il caso che il soggetto conosca entrambi i numeri”. Occorre ricordare che la disgiunzione non traduce un aut aut, dunque è vera anche quando entrambi i termini sono veri (condizione infatti ipoteticamente soddisfacibile anche nel caso in questione). Di conseguenza, sapendo (dai dati sperimentali che abbiamo raccolto) che empiricamente vale B ("è il caso che il soggetto conosca entrambi i numeri"), siamo portati a concludere che il soggetto stia mentendo. Dovendo cioè essere $\neg \mathrm{B}$ falso, secondo la nostra ipotesi non può che valere $\neg \neg A$; così, infine, potremmo concludere che vale A, cioè "il soggetto ha schiacciato entrambi i tasti" e ci ha pertanto mentito.

Il processo logico-deduttivo che abbiamo appena operato potrebbe apparire banale e scontato, la sua logica semplicemente evidente e stringente; eppure - anche accettando di non mettere in dubbio il modello a disposizione per descrivere l'apparato sperimentale - non è l'unico possibile. Fintantoché stipuliamo la verità di $(\neg \neg \mathrm{A} \vee \neg \mathrm{B})$ come ipotesi del nostro modello, per concludere A abbiamo comunque dovuto operare due distinte inferenze logiche; denotando con $\wedge$ l'operatore di congiunzione ("e"), queste sono:

(i) $((\neg \neg \mathrm{A} \vee \neg \mathrm{B}) \wedge \mathrm{B}) \rightarrow \neg \neg \mathrm{A}$.

(ii) $\neg \neg \mathrm{A} \rightarrow \mathrm{A}$.

La regola (i) prende il nome di modus tollendo ponens (o sillogismo disgiuntivo), mentre (ii) rappresenta l'eliminazione della doppia 
negazione. Senza queste due regole di inferenza, non saremmo potuti arrivare alla nostra conclusione sull'esperimento. Avremmo potuto accettare che venisse meno (i) o (ii), e stipulare di ritenere vera l'affermazione del soggetto sperimentale?

L'esperimento mentale sopraesposto è reminiscente del famoso esperimento della doppia fenditura, che all'inizio del XX secolo ebbe un ruolo cruciale per lo sviluppo della Meccanica Quantistica. Proprio come il Gedankenexperiment del mentitore, l'esperimento della doppia fenditura (come anche altri esperimenti di Meccanica Quantistica) pone alcuni problemi interpretativi: gli elettroni attraversano entrambe le fenditure contemporaneamente, solo una, o nessuna?

Di fronte a risultati - sperimentali o teorici - apparentemente paradossali, tipicamente in scienza si considerano due strade: capire se sia stato commesso qualche errore nell'allestimento delle condizioni sperimentali, oppure cambiare la teoria. Una terza possibilità, spesso trascurata, è quella di cambiare sistema logico per la teoria in esame. In effetti, questa via è proprio quella a cui pensarono $G$. Birkhoff e J. von Neumann per tentare di risolvere le problematiche interpretative poste dalla Meccanica Quantistica. Il loro lavoro diede vita ad un sistema formale chiamato Quantum Logic e divergente dalla logica classica [1].

Più in generale, su che base la pratica scientifica adotta o dovrebbe adottare un qualche sistema di regole logico-formali per operare inferenze sulle proposizioni teoriche e sui fenomeni? Che conseguenze ha la scelta di un particolare sistema logico?

\subsection{I processi di inferenza}

La funzione principale della logica è la validazione degli enunciati prescindendo dal loro valore di verità. Ciò significa che la logica non si occupa di operare un controllo riguardo alla verità o falsità delle proposizioni, bensì piuttosto di validare i processi di inferenza, stabilendo se le conclusioni siano correttamente implicate dalle premesse. In breve, il dominio della logica è l'attestazione della correttezza delle inferenze. Per tale ragione, fondamentale importanza nel sistema logico adottato è rivestita sia dalle leggi logiche - enunciati veri per ipotesi, assiomi - sia dalle regole di inferenza - leggi che stabiliamo valere e mediante le quali traiamo conclusioni a partire da enunciati dati (per veri). 
In logica, esistono tre tipi di processi di inferenza distinti. Denotando con P l'antecedente (premessa) e con Q il conseguente (conclusione), si hanno:

a) Deduzione: Q è implicato dall'antecedente $P$ per mezzo di passi formali concatenati, in cui si fa uso di un sistema di regole stabilite.

b) Induzione: $\mathrm{Q}$ è implicato a partire da $\mathrm{P}$ attraverso l'osservazione pregressa che ogni qualvolta valga $\mathrm{P}$, vale anche $\mathrm{Q}$.

c) Abduzione: Q è inferito come miglior spiegazione di P, su una base probabilistica o mediante un processo eminentemente creativo; in virtù di conoscenze pregresse, si considera probabile che stando $\mathrm{P}$, allora valga $\mathrm{Q}$.

L'inferenza deduttiva è pertanto l'unica ad avere carattere di necessità, nel senso che il conseguente segue automaticamente dall'antecedente. Al contrario, le inferenze induttive ed abduttive hanno validità spiccatamente empirica, dal momento che necessitano di un'informazione pregressa e, ciononostante, possono pure essere rese invalide da osservazioni successive.

Un tipico esempio di ragionamento deduttivo è quello della dimostrazione di teoremi in Matematica. Più in generale, si dà un'inferenza deduttiva ogniqualvolta si accettino per vere delle premesse unitamente ad un insieme di regole che ne facciano discendere delle conclusioni. Quest'ultimo punto è molto importante, poiché si comprende come mai non siano ammesse in linea di principio inferenze deduttive per via empirica. Invero, lo stesso sussistere di regole di inferenza non ha affatto carattere di necessità empirica.

In ambito scientifico, la formulazione di teorie si configura come ragionamento abduttivo, anche se l'informazione pregressa su cui valutare il grado di probabilità di una spiegazione si basa su una moltitudine di principi guida, connotati comunque inevitabilmente da un certo grado arbitrarietà (storico, sociale, personale etc...).

\subsection{Il discorso scientifico}

L'inferenza abduttiva è stata considerata il primo passo del ragionamento scientifico [2]. Purtuttavia, come già mostrato, l'uso dell'in- 
ferenza deduttiva è fondamentale ai fini del discorso scientifico. In aggiunta, abbiamo in precedenza sottolineato come cruciale sia l'istituzione di un modello che faccia da ponte tra gli asserti teorici e la realtà fenomenica.

In logica sono stati sviluppati diversi tipi di operatori logici, atti per esempio a modellare situazioni aventi connotazioni modali o temporali, cioè attinenti rispettivamente a categorie di necessità/possibilità o prima/dopo. Tali potenzialità espressive sono fondamentali per il discorso scientifico, poiché senza di esse non potremmo completamente ricondurre le proposizioni teoriche ad un sistema formale. Un'operazione del genere, apparentemente innocua ed anzi necessaria, presenta tuttavia aspetti alquanto delicati e problematici. L'identificazione degli asserti teorici con proposizioni di un sistema formale pone il problema della sovrapposizione tra il piano logico e quello empirico. A questo proposito, si veda ad esempio l'argomento sull'irrealtà del tempo di J.M.E. McTaggart, riportato in [3]. In generale, una condizione necessaria per poter trasferire argomenti logici al piano empirico appare essere una stringente formulazione operazionale dei concetti teorici. Assegnare ad ogni concetto una serie di operazioni - come auspicato da P.W. Bridgman [4] - permette di conferire un significato operativo alle manipolazioni simboliche, con il fine di legittimare la modellazione teorica per mezzo dell'apparato logicoformale adottato.

La disciplina della logica, infine, risulta sufficientemente versatile da trattare questioni di stampo probabilistico e contesti di vaghezza. Da una parte, ciò attiene all'ambito della teoria delle decisioni o della ricerca della miglior spiegazione in ambito scientifico (vd. l'intervento di G.M. D'Ariano). Si consideri come esempio istruttivo la confutazione dell'argomento per cui, dato l'ordine che osserviamo nel mondo, è molto probabile che esista un ente ordinatore [3]. Tramite una trattazione logico-probabilistica, è facile mostrare che, in realtà, l'asserzione precedente equivale ad affermare che è molto probabile che esista, tout court, un ente ordinatore. Dall'altra parte, la Fuzzy Logic è una branca che si occupa di trattare contesti di vaghezza, in cui i predicati non sono nettamente definiti o definibili - un caso molto comune nei discorsi su situazioni quotidiane. In più, sono stati percorsi tentativi di ricondurre l'indeterminatezza e la sovrapposizione quantistiche ad una trattazione di logica fuzzy (per un approccio alternativo ma accostabile, basato sull'istanziazione per gradi, vd. l'intervento di D. Calosi). 


\section{LOGICHE NON CLASSICHE}

Il ragionamento deduttivo operato nel Gedankenexperiment del mentitore fa uso di due regole di inferenza valide in logica classica, ovvero il sillogismo disgiuntivo e l'eliminazione della doppia negazione. Esaminiamo di seguito le conseguenze che possa avere la caduta rispettivamente dell'uno o dell'altro all'interno di un sistema logico.

\subsection{Dialeteismo}

Aristotele considerava il principio di non contraddizione "il più sicuro di tutti i princìpi": irrefutabile, pena l'impossibilità finanche di formulare pensieri [5]. Lo Stagirita problematizzava e attaccava in maniera piuttosto esplicita il pensiero di Eraclito, in particolare la sua dottrina dei contrari. La concezione eraclitea del divenire si fonda sulla coesistenza, negli enti, di aspetti in essenziale opposizione. Secondo Eraclito, concetti irrimediabilmente opposti coabitano ogni ente e sono in esso coessenziali. Il nucleo della critica di Aristotele è il seguente: "È impossibile che la stessa cosa, a un tempo, appartenga e non appartenga a una medesima cosa, secondo lo stesso rispetto. [...] Infatti questi ragionamenti equivalgono, in fondo, a quelli di Eraclito, perché colui che afferma che tutto è vero e tutto è falso, afferma anche separatamente ciascuna di queste dottrine; sicché, se è assurda la dottrina di Eraclito, assurde saranno, anche, queste altre.”.

Il problema di fondo è che, laddove venisse meno il principio di non contraddizione, si andrebbe incontro ad una cosiddetta trivializzazione del sistema logico, per cui qualunque proposizione sarebbe al contempo vera e falsa. La conseguenza di un tale scenario è che un simile sistema logico non avrebbe alcun valore informativo, essendo impossibile formare proposizioni non banali da un punto di vista del valore di verità.

Nella sua accezione logico-semantica, la non-contraddizione si formula come: $\neg(\mathrm{P} \wedge \neg \mathrm{P})$. Come detto, in un sistema di logica classica non è possibile far cadere la non-contraddizione senza andare incontro al cosiddetto principio di esplosione. Infatti, ammettendo anche una sola proposizione contraddittoria nel sistema e usando il sillogismo disgiuntivo, si può dimostrare che qualsiasi proposizione - insieme alla sua negazione - diventa vera (e quindi, al contempo, falsa). Tale proprietà viene riassunta dalla massima: ex falso quodlibet.

Il dialeteismo è un indirizzo filosofico che ammette l'esistenza di con- 
traddizioni vere, cioè di situazioni inconsistenti. Il termine dialeteismo deriva dal prefisso $d i$ - ("duplice") e dal termine greco $\dot{\alpha} \lambda \dot{\eta} \theta \varepsilon i \alpha$ ("verità"). Affinché si possa accettare l'esistenza di alcune proposizioni contraddittorie, senza cadere nella trivializzazione del sistema, bisogna però refutare anche il modus tollendo ponens (utilizzato nell'esperimento mentale del mentitore). Essa non è l'unica legge di logica classica che si usa per derivare il principio di esplosione, ma, a differenza delle altre regole deduttive, questa non è in grado di preservare la verità in presenza di "situazioni inconsistenti" [6]. Si prenda ad esempio la Sylvan's Box [7], una "storia che è inconsistente, ma perfettamente intelligibile". Il dialeteismo presenta anche maggiore versatilità nel trattare situazioni ove il contesto presenti l'ammissibilità di contraddizioni di ordine psicologico-pragmatico. Nell'esperimento mentale del mentitore, lo sperimentatore è forse di fronte a motivi di origine empirica per non accettare l'affermazione del soggetto?

L'approccio dialeteico risolve vari paradossi semantici, come il paradosso del mentitore, di Russell e del sorite. Con le parole di G. Priest: "Tutto il senso della soluzione dialettica ai paradossi semantici sta nell'eliminare la distinzione tra linguaggio oggetto e metalinguaggio." [8].

\subsection{Intuizionismo}

Nel Gedankenexperiment del mentitore abbiamo modellato l'affermazione "non è il caso che il soggetto non abbia schiacciato entrambi i tasti" nella forma $\neg \neg$ P. Sotto una prospettiva di logica classica, abbiamo stipulato l'equivalenza tra $\neg \neg \mathrm{P}$ e P, ed in particolare abbiamo dedotto che "è il caso che il soggetto abbia schiacciato entrambi i tasti" dalla sua doppia negazione. Cosa possiamo dire sull'accettazione di quest'equivalenza? Dire che $\neg \neg \mathrm{P}$ è vera non significa forse affermare che $\neg \mathrm{P}$ è falsa e dunque infine che $\mathrm{P}$, a sua volta, è vera? Dipende.

Se volessimo assegnare ai passi dimostrativi una natura epistemica, la negazione $\neg$ dovrebbe rappresentare una dimostrazione di impossibilità: in altre parole, $\neg \mathrm{P}$ significherebbe che esiste una dimostrazione dell'inesistenza di una dimostrazione di P. Di conseguenza $\neg \neg \mathrm{P}$ ("esiste una dimostrazione che $\neg \mathrm{P}$ non è dimostrabile") non sarebbe equivalente a $\mathrm{P}$, o meglio a "esiste una dimostrazione di P". In questo modo si fa cadere un altro principio cardine della logica classica, il principio del terzo escluso: $\mathrm{P} \vee \neg \mathrm{P}$ (tertium non datur). Tale prospettiva prende il nome di intuizionismo ed è, peraltro, una forma di costruttivismo matematico (cfr. l'intervento di P. Perinotti). 
Senza il principio del terzo escluso non è possibile utilizzare la dimostrazione per assurdo come strumento valido per la deduzione di risultati. Dato un insieme di ipotesi $\mathrm{H}$ ritenute vere, la dimostrazione per assurdo procede assumendo la negazione di ciò che si vuole dimostrare - diciamo $\neg \mathrm{P}-\mathrm{e}$ deducendo una proposizione falsa $\mathrm{Q}$ : in virtù del terzo escluso, si conclude infine P. In simboli: $((\mathrm{H} \wedge \neg \mathrm{P}) \rightarrow \mathrm{Q}) \rightarrow$ $(\mathrm{H} \rightarrow \mathrm{P})$. D'altro canto, un'ottica intuizionista sembra essere particolarmente adatta al fine di dare una qualche valenza epistemica agli asserti teorici: per affermare che un fenomeno sia (non) conoscibile, non basta che sia (non) previsto dalla teoria, bensì deve effettivamente (non) darsi empiricamente. Nell'esperimento mentale del mentitore, lo sperimentatore ha forse avuto evidenza empirica che il soggetto abbia schiacciato entrambi i tasti?

\section{Quale LOGICA, DUNQUe?}

\subsection{La pratica scientifica sotto un'altra logica}

Come detto, l'esperimento mentale descritto all'inizio propone una situazione simile al famoso esperimento della doppia fenditura in Meccanica Quantistica, che rappresenta una realizzazione sperimentale del principio di sovrapposizione quantistico. Storicamente, questo esperimento ha rivestito un ruolo fondamentale per l'avvento e lo sviluppo della Meccanica Quantistica stessa.

Come detto, la Quantum Logic è stato il primo tentativo di abbandonare la logica classica al fine di interpretare una teoria fisica all'apparenza paradossale. Recentemente, sono state persino avanzate proposte di rivedere il principio di sovrapposizione quantistico alla luce della caduta del principio di non contraddizione [9]. A ogni buon conto, sappiamo che la Teoria Quantistica è coerentemente descritta da una teoria di sistemi vestita della usuale teoria della probabilità, che è stato mostrato essere a sua volta un'estensione della logica classica $[10,11]$. Sembra in questo modo profilarsi una sottodeterminazione delle teorie per quanto concerne la logica da adottare.

Quale sarebbe l'interpretazione di un eventuale cambio di logica? La logica, d'altronde, sembra essere più che semplicemente un adeguato e conveniente strumento matematico: soprattutto, un sistema logico porta con sé una particolare Weltanschauung. 


\subsection{Logica scientifica: derivarla o assumerla?}

Per superare il problema della scelta di un sistema logico all'interno della pluralità di quelli anche solo già formulati (senza considerare quelli in linea di principio formulabili), si potrebbe pensare di derivare la logica a posteriori, cioè per via empirica. Per esempio, in questa direzione si configura il famoso lavoro di H. Putnam [12], suggerendo che la logica possa avere statuto empirico proprio alla luce del caso della Teoria Quantistica. In qualche modo, la logica sarebbe scolpita nel modo di manifestarsi dei fenomeni, e dunque potrebbe essere semplicemente conosciuta tramite l'esperienza e universalmente accettata. Tale posizione è stata però criticata, constatando che l'istituzione di un sistema logico è ipso facto un presupposto alla possibilità stessa di interpretare il dominio fenomenico, e non viceversa.

D'altronde, può darsi una seconda via per derivare la logica: mostrare che un dato sistema logico segua necessariamente dalle fondamenta su cui un discorso scientifico si basa. La pratica scientifica, anche debolmente intesa come nell'incipit del presente saggio, assume una serie di precondizioni al proprio stesso concretarsi. Ad esempio, è stato mostrato che un sistema di convenzioni sia sempre necessario (cfr. con l'intervento di L. Poggiali). Si è dunque tentato di rintracciare in un sistema di convenzioni - o più in generale in un qualsiasi insieme di precondizioni necessarie al discorso scientifico - la scaturigine di un dato sistema logico, proponendone pertanto una derivazione deduttiva. Il problema, anche in questo caso, è che un qualche sistema logico è necessario - se non alla formulazione di dette precondizioni, siano esse convenzionali o di altra natura - alla possibilità stessa di operare deduzioni.

Qualsiasi dominio (pertinente alla pratica scientifica) che non attenga a quello strettamente logico, presuppone quest'ultimo come conditio sine qua non o della propria formulabilità, o della possibilità di inferirne alcunché. Di conseguenza, in questo senso il tentativo di dedurre la logica da qualcosa che non sia esso stesso strutturato logicamente è destinato a fallire: c'è bisogno di aver stipulato a priori come procedono le inferenze, per poterne attuare!

Com'è chiaro, in linea di principio si potrebbe ricusare a priori qualsiasi legge logica, addirittura finanche il modus ponens, come nel dialogo What the Tortoise Said to Achilles di L. Carroll [13]. Il modus ponens è una delle regole di inferenza più semplici e basilari di cui solitamente ci avvaliamo: $((\mathrm{P} \rightarrow \mathrm{Q}) \wedge \mathrm{P}) \rightarrow \mathrm{Q}$. La mancata stipula del 
modus ponens, però, porta ad un regressus ad infinitum: pur accettando la premessa $((\mathrm{P} \rightarrow \mathrm{Q}) \wedge \mathrm{P})$, uno potrebbe rifiutare che $((\mathrm{P} \rightarrow \mathrm{Q}) \wedge \mathrm{P})$ $\rightarrow \mathrm{Q}$, e dunque non concludere Q pur stipulando P.

Esattamente come l'apparato di convenzioni che abita ogni teoria, la scelta di un apparato logico-deduttivo sembra avere irredimibilmente carattere di arbitrarietà. Arduo trovare un principio di ragione sufficiente per assumere una qualche regola di inferenza, o un dato assioma. Alcuni principi guida possono essere rintracciati in quelli usuali in scienza, come il Rasoio di Occam, motivazioni di eleganza (argomento tuttavia molto scivoloso), oppure conservativa aderenza - in qualche grado - al sensus communis.

\section{BIBLIOGRAFIA}

[1] Garrett Birkhoff, John Von Neumann, The Logic of Quantum Mechanics, Annals of Mathematics Second Series (1936) Vol. 37, No. 4, pp. 823-843.

[2] Charles Sanders Peirce, Collected Papers of Charles Sanders Peirce, Harvard University Press, Cambridge (USA) (1932).

[3] Graham Priest, Logica, Codice Edizioni, Torino, 2012.

[4] Percy Williams Bridgman, La logica della fisica moderna, Boringhieri, Torino (1965).

[5] Aristotele, Metafisica, Rusconi, Milano, (1994).

[6] Francesco Berto, Lorenzo Bottai, Che cos'è una contraddizione, Carrocci editore, Roma (2015).

[7] Graham Priest, Sylvan's Box: A Short Story and Ten Morals, Notre Dame J. Formal Logic (1997) Vol. 38, No. 4, pp. 573-582.

[8] Bruno Whittle, Dialetheism, logical consequence and hierarchy, Analysis (2004) Vol. 64, No. 4, pp. 318-326.

[9] Newton da Costa, Christian de Ronde, The Paraconsistent Logic of Quantum Superpositions, Foundations of Physics (2013) Vol. 43, No. 7, pp. 845-858.

[10] Edwin Thompson Jaynes, Probability Theory: The Logic of Science, Cambridge University Press, Cambridge (UK) (2003).

[11] Giacomo Mauro D’Ariano, Giulio Chiribella, Paolo Perinotti, Quantum Theory from First Principles: An Informational Approach, Cambridge University Press,Cambridge (UK) (2017).

[12] Hilary Putnam, Is Logic Empirical?, In: Cohen R.S., Wartofsky M.W. (eds) Boston Studies in the Philosophy of Science (1969) Vol. 5, Springer, Dordrecht, pp. 216-241.

[13] Lewis Carroll, What the Tortoise Said to Achilles, Mind (1895) Vol. 104, No. 416, pp. 691-693. 